\title{
Biased random walks on the interlacement set ${ }^{1}$
}

\author{
Alexander Fribergh ${ }^{\mathrm{a}, 2}$ and Serguei Popov ${ }^{\mathrm{b}, 3}$ \\ ${ }^{a}$ Université de Montréal, Department of Mathematics and Statistics, Pavillon André-Aisenstadt 2920, chemin de la Tour, Montréal H3T 1J4, \\ Canada.E-mail:fribergh@dms.umontreal.ca \\ ${ }^{\mathrm{b}}$ Department of Statistics, Institute of Mathematics, Statistics and Scientific Computation, University of Campinas - UNICAMP, rua Sérgio \\ Buarque de Holanda 651, 13083-859, Campinas SP, Brazil.E-mail: popov@ime.unicamp.br
}

Received 19 October 2016; revised 11 April 2017; accepted 24 April 2017

\begin{abstract}
We study a biased random walk on the interlacement set of $\mathbb{Z}^{d}$ for $d \geq 3$. Although the walk is always transient, we can show, in the case $d=3$, that for any value of the bias the walk has a zero limiting speed and actually moves slower than any power.

Résumé. Nous étudions la marche biaisée sur un entrelac aléatoire de $\mathbb{Z}^{d}$ avec $d \geq 3$. Nous montrons que la marche est transiente mais que, dans le cas $d=3$, elle est sous-ballistique pour toutes les valeurs du biais et que ses déplacements sont inférieurs à n'importe quel polynôme.
\end{abstract}

MSC: Primary 60K37; secondary 60G50; 82C41

Keywords: Random walk in random environment; Interlacement set

\section{Introduction}

The model of random interlacements was recently introduced by Sznitman in [21], and detailed accounts can be found in the survey [6] and the recent book [7]. Loosely speaking, random interlacements in $\mathbb{Z}^{d}, d \geq 3$, is a stationary Poissonian soup of (transient) doubly infinite simple random walk trajectories. The level of random interlacements is an additional parameter $u>0$ entering the intensity measure of the Poisson process; as the value of $u$ increases, more trajectories are added. The sites of $\mathbb{Z}^{d}$ that are not touched by the trajectories constitute the vacant set $\mathcal{V}^{u}$ and the union of trajectories is the interlacement set $\mathcal{I}^{u}$, so that $\mathcal{V}^{u}=\mathbb{Z}^{d} \backslash \mathcal{I}^{u}$. It is possible to show that $\mathcal{I}^{u}$ is connected for all $u>0$ a.s., cf. Theorem 1.5 of [5]. In fact, it is possible to construct the random interlacements simultaneously for all $u>0$ in such a way that $\mathcal{V}^{u_{1}} \subset \mathcal{V}^{u_{2}}$ if $u_{1}>u_{2}$. We refer to the above references for the formal definitions (we will give a constructive definition in the next section).

Describing the geometrical properties of the interlacement set can be done in several ways. One of the methods which has been popular in last decade is to study random walks in random environments (RWRE). See [19,20,22] and [2] for further references on the topic. In this paper we aim to do just that by considering the biased random walk on the interlacement set.

This is not the first study of RWRE on the interlacement set. Indeed it has been shown in [14] that the invariance principle holds for the simple random walk on the interlacement cluster; by which we mean that "typical" displacement of the particle by time $n$ is of order $\sqrt{n}$ and that the rescaled process converges to a Brownian motion. This result is similar to what is observed for the simple random walk on the supercritical percolation cluster $[3,12,15]$.

\footnotetext{
${ }^{1}$ Supported by FAPESP (Grant 2012/07166-9).

${ }^{2}$ Supported by the NSERC and FRQNT.

${ }^{3}$ Supported by the CNPq (Grant 300886/2008-0).
} 
In the context of the biased random walk on supercritical percolation clusters in $\mathbb{Z}^{d}$, it has been shown that the walk experiences a phase transition from a positive-speed phase (for small biases) to a zero-speed regime for large biases in which the walk moves as $n^{\gamma}$ for some $\gamma<1$ (see [8]). We show that, for biased random walks on the interlacement set of $\mathbb{Z}^{3}$, the situation is radically different in the sense that for any biases the walk has zero-speed and actually moves slower than any power in $n$.

\subsection{The model}

For $x, y \in \mathbb{Z}^{d}$, let $x \cdot y$ be the usual scalar product, and we denote by $e_{1}, \ldots, e_{d}$ the unit vectors of the canonical orthonormal basis. Also, $\|\cdot\|$ stands for the Euclidean norm. We write $x \sim y$ whenever $\|x-y\|=1$, i.e., $x$ and $y$ are neighbours in $\mathbb{Z}^{d}$.

Let us denote by $P_{x}^{\mathrm{SRW}}$ the law of the $d$-dimensional simple random walk $\left(S_{n}, n \geq 0\right)$ started from $x$.

For any $A \subset \mathbb{Z}^{d}$ let (using the convention $\min \varnothing=+\infty$ )

$$
T_{A}=\min \left\{k \geq 1: S_{k} \in A\right\}
$$

be the hitting time of $A$, and write $T_{x}:=T_{\{x\}}$ for $x \in \mathbb{Z}^{d}$. We define the harmonic measure

$$
e_{A}(x)=P_{x}^{\mathrm{SRW}}\left[T_{A}=\infty\right] \mathbf{1}_{A} ;
$$

the capacity of $A$ is defined by

$$
\operatorname{cap}(A)=\sum_{x \in A} e_{A}(x)
$$

see e.g. Section 6.5 of [10].

Let us give a "constructive" description of random interlacements at level $u$ observed on a finite set $A$. Namely,

- take a Poisson $(u \operatorname{cap}(A))$ number of particles;

- place these particles on the boundary of $A$ independently, with law $\bar{e}_{A}=\left((\operatorname{cap} A)^{-1} e_{A}(x), x \in A\right)$;

- let the particles perform independent simple random walks (by transience, each walk only leaves a finite trace on $A$ ).

As a consequence of the above, we obtain the following useful identity:

$$
\mathbf{P}\left[A \subset \mathcal{V}^{u}\right]=\exp (-u \operatorname{cap}(A)) \text { for all finite } A \subset \mathbb{Z}^{d} .
$$

For fixed $u$, we also define $\mathbf{P}_{0}[\cdot]:=\mathbf{P}\left[\cdot \mid 0 \in \mathcal{I}^{u}\right]$ to be the law of the interlacement set conditioned to contain the origin.

Now, we define the biased random walk on the interlacement cluster, which is the main object of study of this paper. Fix a parameter $\beta>1$ (which accounts for the bias). Let us define the conductances on the edges of $\mathbb{Z}^{d}$ in the following way:

$$
c(x, y)= \begin{cases}\beta^{\max \left(x \cdot e_{1}, y \cdot e_{1}\right)}, & \text { if } x, y \in \mathcal{I}^{u}, x \sim y, \\ 0, & \text { otherwise }\end{cases}
$$

and we call the collection of all conductances $\omega=\left\{c(x, y), x, y \in \mathbb{Z}^{d}\right\}$ the random environment. Consider a random walk $\left(X_{n}, n \geq 0\right)$ in this environment of conductances; i.e., its transition probabilities are given by

$$
q^{\omega}(x, y):=\mathrm{P}^{\omega}\left[X_{n+1}=y \mid X_{n}=x\right]= \begin{cases}\frac{c(x, y)}{\sum_{z} c(x, z)}, & \text { if } x, y \in \mathcal{I}^{u}, x \sim y, \\ 0, & \text { otherwise }\end{cases}
$$

(the superscript in $\mathrm{P}^{\omega}$ indicates that we are dealing with the "quenched" probabilities, i.e., when the underlying graph is already fixed). As usual, we abbreviate $\mathrm{P}_{x}^{\omega}[\cdot]:=\mathrm{P}^{\omega}\left[\cdot \mid X_{0}=x\right]$. For the sake of cleanness, we work under the measure $\mathbf{P}_{0}$; then, we are able to choose the starting point $X_{0}$ to be the origin. Let us also define $\mathbb{P}[\cdot]=\int \mathrm{P}_{0}^{\omega}[\cdot] d \mathbf{P}_{0}$ to be the averaged (a.k.a. annealed) probability measure for the walk starting at the origin. 


\subsection{Results}

The first result is that the random walk is transient.

Theorem 1.1. For $d \geq 3$, we have

$$
\lim _{n \rightarrow \infty}\left\|X_{n}\right\|=\infty, \quad \mathbb{P} \text {-a.s. }
$$

for any fixed drift $\beta>1$ and any intensity parameter $u>0$ of the random interlacements.

Remark 1.2. We believe that $\lim _{n \rightarrow \infty}\left\|X_{n} \cdot e_{1}\right\|=\infty \mathbb{P}$-a.s. for $d \geq 3$. The natural way of proving this would be to adapt the proof of Lemma 1.1 in [18]. However, this proof requires an estimate on the number of left-right crossings (i.e., in the direction $e_{1}$ ) of a large box, which seems to be difficult to obtain in the case of random interlacement (this estimate is used in equation (1.35) of [18]). We decided not to pursue this in this paper, to be able to focus on the surprising behaviour of the speed.

Our main result is that, in three dimensions, the biased random walk on the interlacement cluster has subpolynomial speed:

Theorem 1.3. For $d=3$, we have

$$
\lim _{n \rightarrow \infty} \frac{\ln \left\|X_{n}\right\|}{\ln n}=0, \quad \mathbb{P} \text {-a.s. }
$$

for any fixed drift $\beta>1$ and any intensity parameter $u>0$ of the random interlacements.

As mentioned in the Introduction, this picture is very different from the one we would get by considering the simple random walk on a supercritical percolation cluster in $\mathbb{Z}^{d}$. As will become clear in the course of the proof, the above result is genuinely three-dimensional. Indeed, using the same methods as for our main result, it is possible to show that, in dimensions $d \geq 4$, for large values of the bias the walk still has zero speed (see Theorem 1.4 below); but we conjecture that if $\beta$ is close enough to 1 , then the biased random walk should have positive speed just as in the case of the biased random walk on percolation clusters (see [8]). However, it is unclear how to prove this result because of the difficulties to build a regeneration structure for the walk, due to the lack of the independence property of the environment.

Theorem 1.4. Let $d \geq 4$, and let $\varepsilon>0$ be arbitrary. Then, for all large enough $\beta>1$ (depending on $d$ and $\varepsilon$ ) it holds that

$$
\limsup _{n \rightarrow \infty} \frac{\ln \left\|X_{n}\right\|}{\ln n} \leq \varepsilon, \quad \mathbb{P} \text {-a.s. }
$$

Let us emphasize that, apart from the very last section, this paper is entirely devoted to the case $d=3$.

\section{Preliminary estimates}

We start by introducing some further notation. Given a set $V$ of vertices of $\mathbb{Z}^{d}$ we denote by $|V|$ its cardinality. We define the inner boundary of $V$ as

$$
\partial V=\{x \in V: y \notin V, x \sim y\} .
$$

For any $x \in \mathbb{Z}^{3}$ and $L \geq 1$, we define the ball centered in $x$ and with radius $L$ as

$$
B_{x}(L)=\left\{z \in \mathbb{Z}^{3}:\|z-x\|<L\right\} .
$$

The positive constants (not depending on $n$ ) are denoted by $\gamma, \gamma^{\prime}, \gamma_{1}, \gamma_{2}, \gamma_{3}$ etc. 


\subsection{Connectedness and exit probabilities}

\subsubsection{Exit probabilities of large cones}

For $L_{1,2,3} \geq 0$ let us denote

$$
\operatorname{Cyl}\left(-L_{1}, L_{2}, L_{3}\right)=\left\{z \in \mathbb{Z}^{3},-L_{1} \leq z \cdot e_{1} \leq L_{2},\left|z \cdot e_{i}\right| \leq L_{3} \text { for } i \in\{2,3\}\right\}
$$

and

$$
\partial^{+} \operatorname{Cyl}\left(-L_{1}, L_{2}, L_{3}\right)=\left\{z \in \mathbb{Z}^{3}, z \cdot e_{1}=L_{2},\left|z \cdot e_{i}\right| \leq L_{3} \text { for } i \in\{2,3\}\right\} .
$$

For any $x \in \mathbb{Z}^{3}$, we write $\operatorname{Cyl}_{x}\left(-L_{1}, L_{2}, L_{3}\right)=\left\{y \in \mathbb{Z}^{3}, y=x+z\right.$ with $\left.z \in \operatorname{Cyl}\left(-L_{1}, L_{2}, L_{3}\right)\right\}$ and similarly for $\partial^{+} \operatorname{Cyl}_{x}\left(-L_{1}, L_{2}, L_{3}\right)$.

Lemma 2.1. For any $\alpha \in(0,1]$ there exists $\delta>0$ such that for any fixed $u>0$ and all large enough $n$

$$
\begin{aligned}
& \mathbf{P}_{0}\left[0 \text { is connected to } \partial^{+} \operatorname{Cyl}\left(-n^{\alpha}, n, n^{\alpha}\right) \text { in } \mathcal{I}^{u} \cap \operatorname{Cyl}\left(-n^{\alpha}, n, n^{\alpha}\right)\right] \\
& \quad \geq 1-\exp \left(-\gamma n^{\delta}\right) .
\end{aligned}
$$

Proof. Let $\rho_{u}(x, y)$ be the graph distance (in $\mathcal{I}^{u}$ ) between $x, y \in \mathcal{I}^{u}$, and let $z_{0}=0, z_{1}=k_{1} e_{1}, z=k_{2} e_{1}, \ldots$ be the sites of $\mathcal{I}^{u}$ lying on the ray $\left\{k e_{1}: k \in \mathbb{Z}_{+}\right\}$(where, naturally, $0<k_{1}<k_{2}<\cdots$ ). It holds that the three-dimensional capacity of a "segment" $\left\{k e_{1}: k \in[0, h]\right\}$ is of order $\frac{h}{\ln h}$ (cf. e.g. Proposition 2.4.5 of [9]), so, for fixed $u$ we obtain from (1.2) that

$$
\mathbf{P}_{0}\left[k_{m+1}-k_{m}>h\right] \leq \exp \left(-\gamma^{\prime} \frac{h}{\ln h}\right)
$$

Write, with small enough $\varepsilon>0$

$$
\begin{aligned}
\mathbf{P}_{0} & {\left[\rho_{u}\left(z_{m}, z_{m+1}\right)>s\right] } \\
& \leq \mathbf{P}_{0}\left[k_{m+1}-k_{m}>\varepsilon s\right]+\mathbf{P}_{0}\left[\text { there exists } y \in B_{k_{m} e_{1}}(\varepsilon s) \text { such that } \rho_{u}\left(k_{m} e_{1}, y\right)>s\right] \\
& \leq \exp \left(-\gamma^{\prime} \varepsilon \frac{s}{\ln s}\right)+\gamma^{\prime \prime} \exp \left(-\gamma_{1} s^{\delta}\right),
\end{aligned}
$$

where we used (2.2) to bound the first term and Theorem 1.3 of [5] to bound the second one.

Then, observe that the event in (2.1) contains the event

$$
\left\{\rho_{u}\left(z_{m}, z_{m+1}\right) \leq \frac{n^{\alpha}}{3} \text { for all } m=0, \ldots, n\right\} .
$$

The claim now follows from (2.3) and the union bound.

Define the cone (see Figure 1)

$$
\mathcal{C}_{M}(n)=\left\{x \in \mathbb{Z}^{3},\left|x \cdot e_{1}\right| \leq n,\left|x \cdot e_{i}\right| \leq M\left(n-x \cdot e_{1}\right) \text { for } i \in\{2,3\}\right\},
$$

and its "positive" and "negative" boundaries

$$
\begin{aligned}
& \partial^{-} \mathcal{C}_{M}(n)=\left\{x \in \mathbb{Z}^{3}, x \cdot e_{1}=-n,\left|x \cdot e_{i}\right| \leq M\left(n-x \cdot e_{1}\right) \text { for } i \in\{2,3\}\right\}, \\
& \partial^{+} \mathcal{C}_{M}(n)=\partial \mathcal{C}_{M}(n) \backslash \partial^{-} \mathcal{C}_{M}(n) .
\end{aligned}
$$

First, we prove that it is very probable that the walker exits large cones on the positive side: 


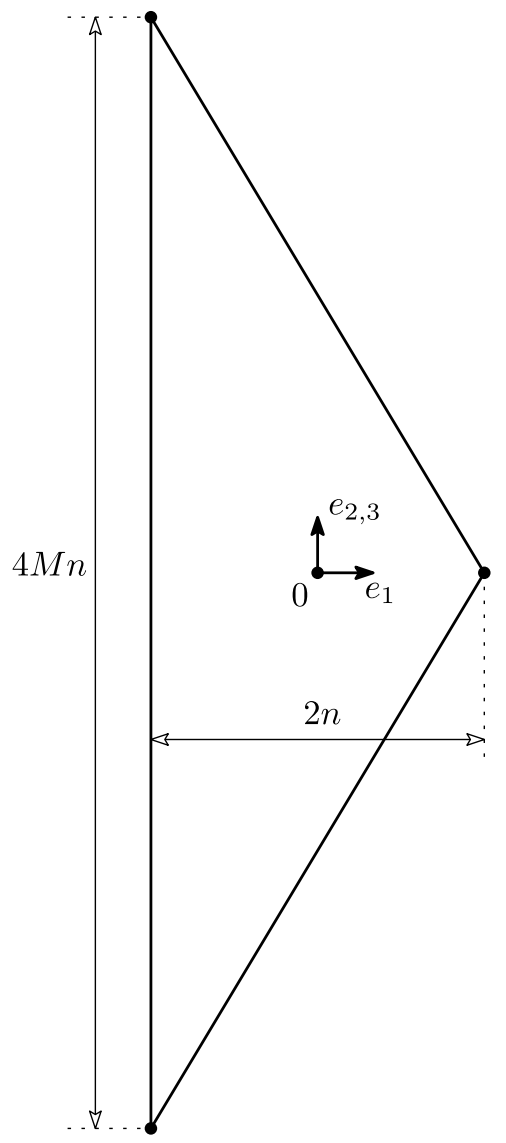

Fig. 1. The cone $\mathcal{C}_{M}(n)$.

Lemma 2.2. For any $M<\infty$, we have

$$
\mathbb{P}\left[T_{\partial}-\mathcal{C}_{M}(n)<T_{\partial}+\mathcal{C}_{M}(n)\right] \leq \gamma \exp \left(-\gamma n^{\delta}\right),
$$

for some $\delta<1$.

Proof. Let us consider the event

$$
G_{n}:=\left\{0 \text { is connected in } \mathcal{I}^{u} \cap \operatorname{Cyl}\left(-n^{\alpha}, n, n^{\alpha}\right) \text { to } \partial^{+} \operatorname{Cyl}\left(-n^{\alpha}, n, n^{\alpha}\right)\right\} .
$$

For any environment $\omega$, we may use equation (4) in [4] (or also exercise 2.36 in [11]) to see that

$$
P_{0}^{\omega}\left[T_{\partial}-\mathcal{C}_{M}(n)<T_{\partial}+\mathcal{C}_{M}(n)\right] \leq \frac{C^{\omega}\left(0 \leftrightarrow \partial^{-} \mathcal{C}_{M}(n)\right)}{C^{\omega}\left(0 \leftrightarrow \partial \mathcal{C}_{M}(n)\right)},
$$

where $C^{\omega}$ stands for the effective conductance in the (weighted) interlacement graph restricted on $\mathcal{C}_{M}(n)$. In an environment $\omega \in G_{n}$, we know that there is a simple path from 0 to $\partial^{+} \operatorname{Cyl}\left(-n^{\alpha}, n, n^{\alpha}\right)$ within $\operatorname{Cyl}\left(-n^{\alpha}, n, n^{\alpha}\right)$ and because of our definition of $\mathcal{C}_{M}(n)$ this path has to cross $\partial \mathcal{C}_{M}(n)$ before reaching $\partial^{+} \operatorname{Cyl}\left(-n^{\alpha}, n, n^{\alpha}\right)$. This implies that for $\omega \in G_{n}$ there exists a path $\mathcal{P}$, composed of $v_{0}=0, v_{1}, \ldots, v_{i_{0}}$ such that

(1) $v_{i_{0}} \in \partial \mathcal{C}_{M}(n)$,

(2) $i_{0} \leq\left|\operatorname{Cyl}\left(-n^{\alpha}, n, n^{\alpha}\right)\right| \leq \gamma n^{3}$,

(3) $v_{j} \cdot e_{1} \geq-n^{\alpha}$ for any $j \leq i_{0}$. 
We recall that Rayleigh's monotonicity principle (see Section 2.4 of [11]) implies that closing edges in a graph decreases all effective conductances. Hence, we know that the effective conductance $C^{\omega}\left(0 \leftrightarrow \partial \mathcal{C}_{M}(n)\right)$ can be lower bounded by the conductance of the path $\mathcal{P}$ which is at least $c n^{-3} \beta^{-n^{\alpha}}$ (where $\alpha<1$ ) since it is composed of at most $\gamma n^{3}$ edges in series which have conductance at least $c \beta^{-n^{\alpha}}$. Hence, in an environment $\omega$ belonging to the event appearing in Lemma 2.1, we have

$$
C^{\omega}\left(0 \leftrightarrow \partial \mathcal{C}_{M}(n)\right) \geq c n^{-3} \beta^{-n^{\alpha}} .
$$

Rayleigh's monotonicity principle also implies that merging vertices together increases effective conductances. Let us merge all vertices of $\mathcal{C}_{M}(n) \backslash \partial^{-} \mathcal{C}_{M}(n)$ (which contains the origin) into $\Delta_{1}$ and all vertices of $\partial^{-} \mathcal{C}_{M}(n)$ into $\Delta_{2}$; we can use Rayleigh's monotonicity principle to see that $C^{\omega}\left(0 \leftrightarrow \partial^{-} \mathcal{C}_{M}(n)\right) \leq C^{\omega}\left(\Delta_{1} \leftrightarrow \Delta_{2}\right)$. The latter can be upper bounded by seeing that $\Delta_{1}$ and $\Delta_{2}$ are linked by at most $\gamma n^{3}$ edges of conductances at most $\gamma \beta^{-n}$. Hence

$$
C^{\omega}\left(0 \leftrightarrow \partial^{-} \mathcal{C}_{M}(n)\right) \leq \gamma n^{3} \beta^{-n} .
$$

Putting together (2.5), (2.6) and (2.7) we see that, for any $\omega$ belonging to the event appearing in Lemma 2.1, we have

$$
\mathrm{P}_{0}^{\omega}\left[T_{\partial}-\mathcal{C}_{M}(n)<T_{\partial}+\mathcal{C}_{M}(n)\right] \leq \gamma n^{6} \beta^{-n+n^{\alpha}},
$$

for some $\alpha<1$. Hence, by using Lemma 2.1, we see that for some $\delta<1$ we have

$$
\begin{aligned}
\mathbb{P}\left[T_{\partial}-\mathcal{C}_{M}(n)<T_{\partial}+\mathcal{C}_{M}(n)\right. & =\mathbb{P}\left[G_{n}^{c}\right]+\mathbf{E}\left[G_{n}, \mathrm{P}_{0}^{\omega}\left[T_{\partial}-\mathcal{C}_{M}(n)<T_{\partial}+\mathcal{C}_{M}(n)\right]\right] \\
& \leq \gamma^{\prime} \exp \left(-\gamma n^{\delta}\right),
\end{aligned}
$$

which implies the result.

Let us introduce

$$
\Phi_{n}=\bigcap_{i=1}^{n^{2 / 3}}\left\{T_{\partial+\mathcal{C}_{M}\left(i n^{1 / 3}\right)}<T_{\partial-\mathcal{C}_{M}\left(i n^{1 / 3}\right)}\right\} ;
$$

we can then use the union bound and Lemma 2.2 to see that

$$
\mathbb{P}\left[\Phi_{n}^{c}\right] \leq \gamma^{\prime} \sum_{i=1}^{n^{2 / 3}} \exp \left(-\gamma\left(i n^{1 / 3}\right)^{\delta}\right) .
$$

\subsubsection{Proof of transience}

Let us define

$$
K(n)=\left\{x \in \mathbb{Z}^{3}, x \cdot e_{1}>-n,\left|x \cdot e_{i}\right| \leq n+x \cdot e_{1} \text { for } i \in\{2,3\}\right\} .
$$

Lemma 2.3. There exists $\delta>0$ such that for any fixed $u>0$ and for all $n$ large enough

$$
\mathbf{P}_{0}\left[0 \text { is connected to infinity in } \mathcal{I}^{u} \cap K(n)\right] \geq 1-\exp \left(-\gamma n^{\delta}\right) .
$$

Proof. This can be proved quite similarly to Lemma 2.1. Using the same notation, observe that the event in (2.10) contains the event

$$
\left\{\rho_{u}\left(z_{m}, z_{m+1}\right) \leq \frac{n+m}{3} \text { for all } m \geq 0\right\} .
$$

Again, the claim follows from (2.3) and the union bound. 


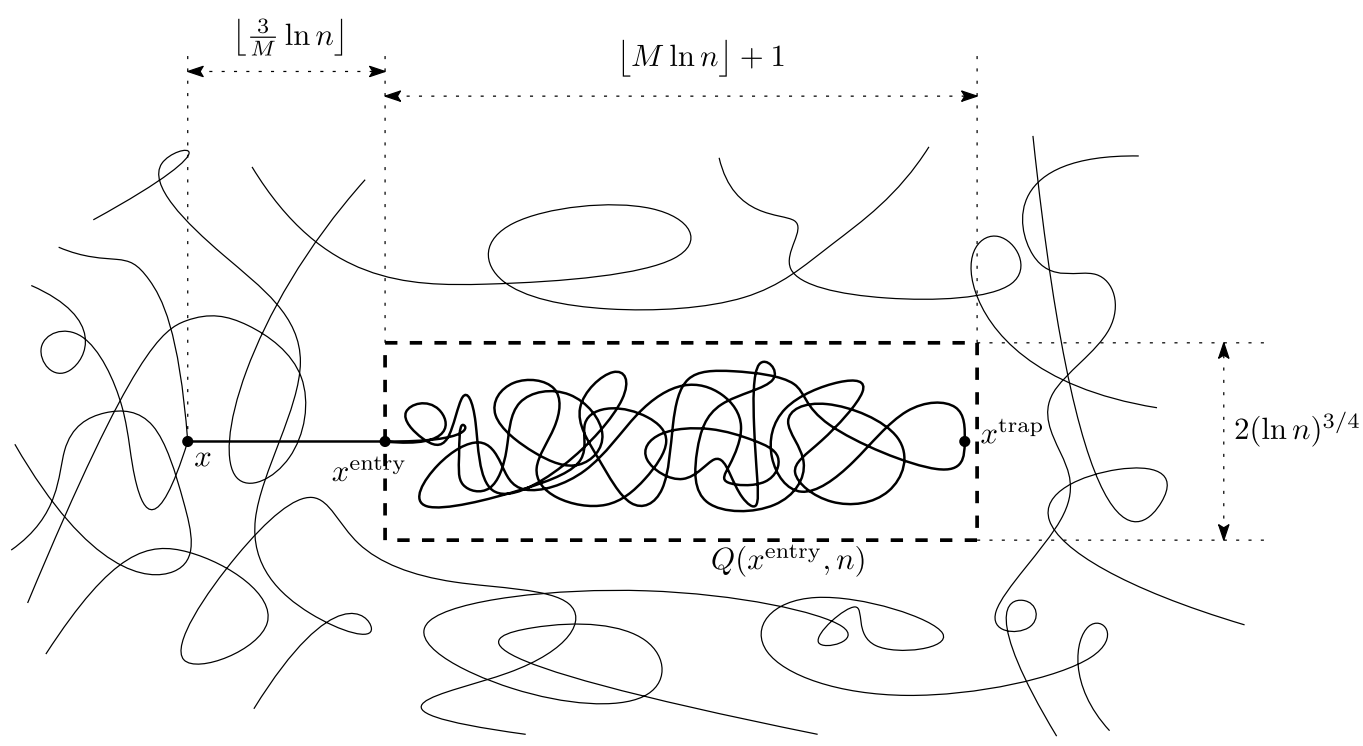

Fig. 2. A trap for the random walk $X$. The solid lines/curves are the interlacements; the trajectory of the RWRE $X$ is not shown on the picture.

Let us now prove Theorem 1.1.

Proof. Using Lemma 2.3 and Borel-Cantelli we can show that $\mathbf{P}_{0}$-a.s. there exists $N(\omega)$ such that 0 belongs to an infinite simple path lying in $\mathcal{I}^{u} \cap K(N(\omega))$.

For any $k \geq 0$, this path will contain at most $\gamma k^{2}$ edges of conductances $\gamma \beta^{k-N(\omega)+1}$. This means, using Rayleigh's monotonicity principle and the law of resistances in series (see Chapter 2 of [11]), we can prove that

$$
R^{\omega}(0 \leftrightarrow \infty) \leq \gamma \sum_{k \geq 0} k^{2} \beta^{N(\omega)-k+1}<\infty .
$$

This means that the resistance from 0 to $\infty$ is finite, which means that the random walk is transient (see Chapter 2 of [11]).

\subsection{Traps}

Let us remind the reader that we are working in three dimensions.

As usual, the method for proving that the biased random walk has zero speed is showing that it will encounter a trap, i.e., a part of the environment where the random walk will stay for a long time. For a biased random walk, this consists in looking for dead-ends in the direction $e_{1}$ from, once the biased walk has entered such a dead-end, it will have to fight against the drift to exit the trap.

From now on we assume that $M$ is not too small, say, $M \geq 10$. Let us introduce the "quiver" set

$$
Q(x, M, n)=\partial \mathrm{Cyl}_{x}\left(0,\lfloor M \ln n\rfloor+1,(\ln n)^{3 / 4}\right),
$$

where $\lfloor\cdot\rfloor$ stands for the integer part. Also, for $x \in \mathbb{Z}^{3}$ we denote $x^{\text {entry }}=x^{\text {entry }}(x, M, n)=x+\left\lfloor\frac{3}{M} \ln n\right\rfloor e_{1}$ and $x^{\text {trap }}=$ $x^{\text {trap }}(x, M, n)=x^{\text {entry }}+\lfloor M \ln n\rfloor e_{1}$. Observe that $x^{\text {entry }} \in Q\left(x^{\text {entry }}, M, n\right)$ and $x^{\text {trap }}$ is strictly inside $Q\left(x^{\text {entry }}, M, n\right)$.

Our goal is to find a trapping structure for the walk. Let us denote $\mathcal{T}(x, M, n)$ the event that there exists a trap at $x$ (see Figure 2), defined in the following way: let

$$
\begin{aligned}
& \mathcal{T}^{(1)}(x, M, n)=\left\{\mathcal{I}_{u} \text { intersects } Q\left(x^{\text {entry }}, M, n\right) \text { only at } x^{\text {entry }},\right. \\
& \mathcal{T}^{(2)}(x, M, n)=\left\{x+j e_{1} \in \mathcal{I}_{u}, \text { for all } 0 \leq j \leq\left\lfloor\frac{3}{M} \ln n\right\rfloor\right\},
\end{aligned}
$$




$$
\mathcal{T}^{(3)}(x, M, n)=\left\{x^{\text {trap }} \in \mathcal{I}_{u}\right\},
$$

and we define $\mathcal{T}(x, M, n)=\mathcal{T}^{(1)}(x, M, n) \cap \mathcal{T}^{(2)}(x, M, n) \cap \mathcal{T}^{(3)}(x, M, n)$.

Not focusing, for now, on technicalities, the key part of the event $\mathcal{T}(x, M, n)$ is that, not far away from $x$ in the direction of the drift, there is a structure in $\mathcal{I}_{u}$ creating a dead end for the biased random walk.

Such a structure can appear if, for example

(1) all the walk traces forming $\mathcal{I}_{u}$ except one avoid $Q\left(x^{\text {entry }}, M, n\right)$;

(2) simultaneously, the one remaining walk's trace has a behavior such that the two last conditions present in the definition of $\mathcal{T}(x, M, n)$ are satisfied.

These two types of events are the ones we are going to study in order to understand the likelihood of finding traps.

Recall that $P_{x}^{\mathrm{SRW}}$ stands for the law of simple random walk $\left(S_{n}, n \geq 0\right)$ started from $x$. Let $\sigma_{1}=\min \left\{k>T_{x}\right.$ entry : $\left.S_{k}=x^{\text {trap }}\right\}$ be the time of the first visit to $x^{\text {trap }}$ after visiting $x^{\text {entry }}, \sigma_{2}=\min \left\{k>T_{x}\right.$ entry $\left.: S_{k}=x^{\text {entry }}\right\}$ be the moment when $x^{\text {entry }}$ is visited for the second time, and $\sigma_{3}=\min \left\{k>T_{x}\right.$ entry $\left.: S_{k} \in Q\left(x^{\text {entry }}, M, n\right)\right\}$ be the moment when $Q\left(x^{\text {entry }}, M, n\right)$ is first visited after $T_{x}$ entry. Define the event

$$
\begin{aligned}
E_{x, M, n}= & \left\{S_{i}=x+i e_{1}, i=1, \ldots,\left\lfloor\frac{3}{M} \ln n\right\rfloor, \sigma_{1}<\sigma_{2}=\sigma_{3},\right. \\
& \left.S_{\sigma_{2}+j}=x^{\text {entry }}-j e_{1}, j=1, \ldots,\left\lfloor\frac{3}{M} \ln n\right\rfloor\right\} ;
\end{aligned}
$$

that is, the trajectory makes a loop that first goes straight from $x$ to $x^{\text {entry }}$, then goes to $x^{\text {trap }}$ and returns to $x^{\text {entry }}$ strictly inside $Q\left(x^{\text {entry }}, M, n\right)$, and then returns straight to $x$, see Figure 2.

Lemma 2.4. There is a constant $\gamma>0$ such that

$$
P_{x}^{\mathrm{SRW}}\left[E_{x, M, n}\right] \geq n^{-\gamma / M}
$$

for all large enough $n$.

Proof. Clearly, it holds that

$$
P_{x}^{\mathrm{SRW}}\left[S_{j}=x+j e_{1}, S_{\sigma_{2}+j}=x^{\mathrm{entry}}-j e_{1}, j=1, \ldots,\left\lfloor\frac{3}{M} \ln n\right\rfloor\right]=6^{-2\left\lfloor\frac{3}{M} \ln n\right\rfloor},
$$

so it remains to find a lower bound on the probability that the trajectory behaves as it should inside $Q\left(x^{\text {entry }}, M, n\right)$.

Define

$$
\begin{aligned}
& \sigma_{1}^{\prime}=\min \left\{k \geq 1: S_{k} \cdot e_{1}=x^{\text {entry }} \cdot e_{1}+\lfloor M \ln n\rfloor-\left\lfloor\frac{1}{M} \ln n\right\rfloor\right\} \\
& \sigma_{2}^{\prime}=\min \left\{k \geq 1: S_{k} \cdot e_{1}=x^{\text {entry }} \cdot e_{1}+\left\lfloor\frac{1}{M} \ln n\right\rfloor\right\} .
\end{aligned}
$$

Then, by the (strong) Markov property it holds that (recall that $x^{\text {entry }} \in Q\left(x^{\text {entry }}, M, n\right)$ )

$$
\begin{aligned}
P_{x^{\text {entry }}}^{\mathrm{SRW}} & \left.T_{x^{\text {trap }}}<T_{x^{\text {entry }}}=T_{Q\left(x^{\text {entry }}, M, n\right)}\right] \\
\geq & P_{x^{\text {entry }}}^{\mathrm{SRW}}\left[\sigma_{1}^{\prime}<T_{Q\left(x^{\text {entry }}, M, n\right)},\left(S_{1}-x^{\text {entry }}\right) \cdot e_{1}=1\right] \\
& \times P_{x^{\text {entry }}}^{\mathrm{SRW}}\left[T_{x^{\text {trap }}}-\sigma_{1}^{\prime} \leq 3\left\lfloor\frac{1}{M} \ln n\right\rfloor<T_{Q\left(x^{\text {entry }}, M, n\right)} \mid\left(S_{1}-x^{\text {entry }}\right) \cdot e_{1}=1, \sigma_{1}^{\prime}<T_{Q\left(x^{\text {entry }}, M, n\right)}\right] \\
& \times P_{x^{\text {trap }}}^{\mathrm{SRW}}\left[\sigma_{2}^{\prime}<T_{Q\left(x^{\text {entry }}, M, n\right)}\right]
\end{aligned}
$$




$$
\begin{aligned}
& \times P_{x^{\text {trap }}}^{\mathrm{SRW}}\left[T_{x^{\text {entry }}}-\sigma_{2}^{\prime} \leq 3\left\lfloor\frac{1}{M} \ln n\right\rfloor<T_{Q\left(x^{\text {entry }}, M, n\right)} \mid \sigma_{2}^{\prime}<T_{Q\left(x^{\text {entry }}, M, n\right)}\right] \\
& =: F_{1} \times F_{2} \times F_{3} \times F_{4} \text {, }
\end{aligned}
$$

$F_{2} \wedge F_{4} \geq 6^{-3\left\lfloor\frac{1}{M} \ln n\right\rfloor}$

(just follow a fixed path of length at most $3\left\lfloor\frac{1}{M} \ln n\right\rfloor$ that leads to $x^{\text {entry }}$ or $x^{\text {trap }}$ ).

In order to estimate the other two terms in (2.15), denote $\tilde{S}_{k}^{(i)}=S_{k} \cdot e_{i}, i=1,2,3$ and

$$
S_{k}^{(i)}=\tilde{S}_{\theta_{k}^{(i)}}^{(i)} \quad \text { with } \theta_{k}^{(i)}=\inf \left\{j>\theta_{k-1}^{(i)}, \tilde{S}_{j}^{(i)}-\tilde{S}_{j-1}^{(i)} \neq 0\right\}
$$

initialized with $\theta_{0}^{(i)}=0$. In words, $S_{k}^{(i)}$ records the successive steps of the SRW $S$ in the $i$ th direction. Obviously, we have that $S^{(1)}, S^{(2)}$ and $S^{(3)}$ are independent (the same is not true for $\tilde{S}^{(i)}$ ).

Let $T^{(i)}$ 's be the corresponding hitting times defined analogously to (1.1). We then write

$$
\begin{aligned}
P_{0}^{\mathrm{SRW}} & {\left[T_{\lfloor M \ln n\rfloor-\left\lfloor\frac{1}{M} \ln n\right\rfloor}^{(1)}<M^{2} \ln ^{2} n, T_{\lfloor M \ln n\rfloor-\left\lfloor\frac{1}{M} \ln n\right\rfloor}^{(1)}<T_{0}^{(1)}\right] } \\
= & P_{0}^{\mathrm{SRW}}\left[T_{\lfloor M \ln n\rfloor-\left\lfloor\frac{1}{M} \ln n\right\rfloor}^{(1)}<M^{2} \ln ^{2} n \mid T_{\lfloor M \ln n\rfloor-\left\lfloor\frac{1}{M} \ln n\right\rfloor}^{(1)}<T_{0}^{(1)}\right] \\
& \times P_{0}^{\mathrm{SRW}}\left[T_{\lfloor M \ln n\rfloor-\left\lfloor\frac{1}{M} \ln n\right\rfloor}^{(1)}<T_{0}^{(1)}\right] .
\end{aligned}
$$

Clearly, the second term in the right-hand side of the above display is bounded below by $1 /\lfloor M \ln n\rfloor$. As for the first term, observe that formula (6) of [17] implies that the conditional expectation of the time the simple random walk starting at 1 hits $a \geq 2$, given that it hits $a$ before hitting 0 , is equal to $\left(a^{2}-1\right) / 3$. So, using Chebyshev's inequality for the probability of the complementary event, we see that the first term is bounded below by $2 / 3$. Therefore, we obtain that

$$
P_{0}^{\mathrm{SRW}}\left[T_{\lfloor M \ln n\rfloor-\left\lfloor\frac{1}{M} \ln n\right\rfloor}^{(1)}<M^{2} \ln ^{2} n, T_{\lfloor M \ln n\rfloor-\left\lfloor\frac{1}{M} \ln n\right\rfloor}^{(1)}<T_{0}^{(1)}\right] \geq \frac{2}{3 M \ln n},
$$

which loosely speaking means that the first component has probability at least $\frac{2}{3 M \ln n}$ to reach the right-hand side of the quiver in time a time less than $M^{2} \ln ^{2} n$. Also, denote

$$
\tau^{(i)}=\min \left\{k:\left|S_{k}^{(i)}\right|=\left\lfloor(\ln n)^{3 / 4}\right\rfloor\right\}
$$

for $i=2,3$. It holds that (see e.g. $\$ 21$ of Chapter V of [16])

$$
P^{\mathrm{SRW}}\left[\tau^{(i)} \geq \frac{1}{4} M^{2} \ln ^{2} n\right] \geq \exp \left(-\gamma^{\prime \prime} M^{2}(\ln n)^{1 / 2}\right),
$$

this means that the second and third coordinates have probability at least $\exp \left(-\gamma^{\prime \prime} M^{2}(\ln n)^{1 / 2}\right)$ to stay confined in the quiver for a time at least $\frac{1}{4} M^{2} \ln ^{2} n$.

The last remaining step is to notice that by the law of large numbers the time-changes $\theta^{(i)}$ are such that

$$
P^{\mathrm{SRW}}\left[\theta_{M^{2} \ln ^{2} n}^{(1)} \leq \frac{1}{4} M^{2} \ln ^{2} n, \theta_{\frac{1}{4} M^{2} \ln ^{2} n}^{(2)}>\frac{1}{8} M^{2} \ln ^{2} n, \theta_{\frac{1}{4} M^{2} \ln ^{2} n}^{(3)}>\frac{1}{8} M^{2} \ln ^{2} n\right] \geq \frac{1}{2},
$$

since asymptotically $1 / 3$ of the steps should be taken in any directions.

Since the time changes are independent of the walks $S^{(j)}$ we can use (2.17) and (2.18) to obtain that

$$
F_{1} \wedge F_{3} \geq \frac{1}{2} \times \frac{2}{3 M \ln n} \times\left(\frac{\gamma^{\prime} \exp \left(-\gamma^{\prime \prime}(\ln n)^{1 / 2}\right)}{M \ln n}\right)^{2}
$$

and this concludes the proof of Lemma 2.4. 


\subsection{Finding traps in the interlacement set}

First of all, we need to estimate the "cost" of having a trap in some fixed place.

Lemma 2.5. In three dimensions, we have $\operatorname{cap}(Q(x, M, n)) \leq \frac{\gamma M \ln n}{\ln \ln n}$.

Proof. Abbreviate, for now, $Q:=Q(x, M, n)$ and $m:=\ln n$. Also, let $g(x, y)$ be the Green's function of the simple random walk; it is well known that for all $x, y \in \mathbb{Z}^{3}$

$$
g(x, y)=g(y, x)=g(0, y-x) \leq \frac{\hat{\gamma}}{1+\|x-y\|}
$$

for some positive constant $\hat{\gamma}$. Let us define the set of functions

$$
\Sigma^{\downarrow}=\left\{\varphi \in \mathbb{R}^{\mathbb{Z}^{3}}: \sum_{y \in Q} g(x, y) \varphi(y) \geq 1 \text { for all } x \in Q\right\} .
$$

Then it holds that

$$
\operatorname{cap}(Q)=\inf _{\varphi \in \Sigma^{\downarrow}} \sum_{x \in Q} \varphi(x),
$$

see Lemma 1.14 of [7].

Now, it is elementary to observe that for any $x \in Q$

$$
|\{y \in Q:\|x-y\| \in[k, k+1)\}| \geq \begin{cases}\gamma_{1} k, & \text { for } k=1, \ldots,\left\lfloor m^{3 / 4}\right\rfloor \\ \gamma_{2} m^{3 / 4}, & \text { for } k=\left\lfloor m^{3 / 4}\right\rfloor+1, \ldots, \frac{1}{2} M m .\end{cases}
$$

From this, for any $x \in Q$ we obtain

$$
\begin{aligned}
\sum_{y \in Q} \frac{1}{\|x-y\|} & \geq \sum_{k=1}^{\frac{1}{2} M m} \frac{|\{y:\|x-y\| \in[k, k+1)\}|}{k} \\
& \geq \gamma_{1} m^{3 / 4}+\gamma_{2} \sum_{k=\left\lfloor m^{3 / 4}\right\rfloor+1}^{\frac{1}{2} M m} \frac{m^{3 / 4}}{k} \\
& \geq \gamma_{3} m^{3 / 4} \ln m,
\end{aligned}
$$

so (recall (2.20)) a function $\varphi$ that equals $\frac{\gamma_{4}}{m^{3 / 4} \ln m}$ on $Q$ for large enough $\gamma_{4}$, belongs to $\Sigma^{\downarrow}$ for all $n$ large enough. The claim of Lemma 2.5 now follows from (2.21) since $|Q|$ is of order $M m^{7 / 4}$.

One technical difficulty in proving that we regularly encounter traps is that we want to take into account the information obtained from the past trajectory of the walk; indeed, correlations in the interlacement set have infinite range. That is, we need to be able to work with the conditional law of the interlacement set, given that inside some finite set the interlacement configuration is (partially or even completely) revealed. Next, we formulate a result from [1] about the conditional decoupling for random interlacements. We also observe that the unconditional decoupling from [13] is not enough in this situation.

With some abuse of notation, we denote by $\mathcal{I}_{A}^{u}$ the interlacement configuration on level $u$ restricted on $A$, i.e., for $x \in A$ we write $\mathcal{I}_{A}^{u}(x)=1$ whenever $x \in \mathcal{I}^{u}$. 
Proposition 2.6. Let $u^{\prime}>u>0$, and let $A_{1}=B_{0}(r), A_{2} \subset \mathbb{Z}^{3} \backslash B_{0}(r+s)$; assume that $\gamma_{1} s \leq r \leq \gamma_{2}$ s for some fixed $\gamma_{1,2}>0$. Then, there are positive constants $\gamma, \gamma^{\prime}$ depending only on dimension, and a (measurable) set $\mathcal{G}_{u^{\prime}} \in\{0,1\}^{A_{2}}$ such that

$$
\mathbf{P}\left[\mathcal{I}_{A_{2}}^{u} \in \mathcal{G}_{u^{\prime}}\right] \geq 1-\exp \left(-\gamma^{\prime} u^{\prime} s^{d-2}\right),
$$

and for any increasing event $E$ on the interlacements set intersected with $A_{1}$, we have

$$
\begin{aligned}
& \mathbf{P}\left[E\left(\mathcal{I}_{A_{1}}^{u}\right) \mid \mathcal{I}_{A_{2}}^{u}\right] \mathbf{1}\left\{\mathcal{I}_{A_{2}}^{u} \in \mathcal{G}_{u^{\prime}}\right\} \\
& \quad \leq\left(\mathbf{P}\left[E\left(\mathcal{I}_{A_{1}}^{u+u^{\prime}}\right)\right]+\gamma \exp \left(-\gamma^{\prime} u^{\prime} s^{d-2}\right)\right) \mathbf{1}\left\{\mathcal{I}_{A_{2}}^{u} \in \mathcal{G}_{u^{\prime}}\right\} .
\end{aligned}
$$

Proof. This is an immediate corollary of Theorem 2.2 of [1].

The above decoupling result implies the following:

Lemma 2.7. For any $M$ there exists a constant $g_{M}>0$ and a set $\mathcal{G}(x, M, n)$ of good environments on $\mathcal{C}_{M}(n)$ such that $\mathbf{P}_{0}[\mathcal{G}(x, M, n)] \geq 1-n^{-12}$ and, for $\mathcal{I}_{\mathcal{C}_{M}(n)}^{u} \in \mathcal{G}(x, M, n)$

$$
\mathbf{P}_{0}\left[Q\left(x^{\text {entry }}, M, n\right) \subset \mathcal{V}^{u} \mid \mathcal{I}_{\mathcal{C}_{M}(n)}^{u}\right] \geq \exp \left(-\frac{g_{M} \ln n}{\ln \ln n}\right)
$$

for any $x \in \partial^{+} \mathcal{C}_{M}(n)$

Proof. Indeed, let us first note that one can insert $Q\left(x^{\text {entry }}, M, n\right)$ inside a ball of radius $M \ln n$ in such a way that the distance between this ball and $\mathcal{C}_{M}(n)$ would be at least $\frac{2}{M} \ln n$.

Then, use Proposition 2.6 with the increasing event $\left\{Q\left(x^{\text {entry }}, M, n\right) \cap \mathcal{I}^{u} \neq \varnothing\right\}, r=M \ln n$ and $s=\frac{2}{M} \ln n$.

Observe that one can choose a large enough $u^{\prime}$ in such a way that the probability of the event $(\mathcal{G}(x, M, n))^{\complement}$ would be bounded above by any negative power of $n$.

An application of (1.2) together with Lemma 2.5 yields that for $\mathcal{I}_{\mathcal{C}_{M}(n)}^{u} \in \mathcal{G}(x, M, n)$

$$
\mathbf{P}_{0}\left[Q\left(x^{\text {entry }}, M, n\right) \cap \mathcal{I}^{u} \neq \varnothing \mid \mathcal{I}_{\mathcal{C}_{M}(n)}^{u}\right] \leq 1-\exp \left(-\frac{g_{M} \ln n}{\ln \ln n}\right)+C n^{-12},
$$

which finishes the proof.

The second important part for constructing a trap is to find an interlacement that actually creates the trap inside the cylinder avoided by all the other walks. This is our aim for now. We need a result about "adding a loop to an existing configuration", which we now describe. Let $A$ be a finite subset of $\mathbb{Z}^{d}, d \geq 3$ (we formulate this result for general $d$ since it may be of independent interest). Fix any $x_{0} \in A$ and let $x_{0}=y_{0} \sim y_{1} \sim \ldots \sim y_{m}=x_{0}$ be a nearest-neighbour path that begins and ends in $x_{0}$ and such that $y_{k} \in A \backslash \partial A$ for $k=0, \ldots, m$. In the result below we need a finer control of the random interlacements: let $\mathcal{L}^{u}(x)$ be the local time at site $x$ at level $u$, that is, the sum of local (occupation) times in $x$ of all trajectories at level $u$. We denote by $\eta \in\{0,1,2, \ldots\}{ }^{A}$ a generic configuration of local times on $A$, and by $\ell$ the configuration "generated" by the above loop, i.e., $\ell(x)=\sum_{k=1}^{m} \mathbf{1}\left\{y_{k}=x\right\}$. Write $(\eta+\ell)(x):=\eta(x)+\ell(x)$, and denote by $\mathcal{L}_{A}^{u}(x)$ the local time configuration on $A \subset \mathbb{Z}^{d}$.

Lemma 2.8. For any $\eta$ such that $\eta\left(x_{0}\right)=1$ we have

$$
\mathbf{P}\left[\mathcal{L}_{A}^{u}=\eta+\ell\right] \geq(2 d)^{-m} \mathbf{P}\left[\mathcal{L}_{A}^{u}=\eta\right]
$$

(recall that $x_{0}$ is the initial vertex of the loop, and $m$ is the number of steps in the loop). 
Proof. A trace on $A$ is a finite sequence $v=\left(v_{0}, \ldots, v_{s}\right)$ of vertices of $A$ such that either $v_{i-1} \sim v_{i}$ or $v_{i-1}, v_{i} \in \partial A$ for all $i=1, \ldots, s$, and also $v_{0}, v_{s} \in \partial A$. For a trace $v$, define its weight as

$$
p_{v}=\prod_{j=1}^{s} P_{v_{j-1}}^{\mathrm{SRW}}\left[T_{A}<\infty, S_{T_{A}}=v_{j}\right] \times P_{v_{s}}^{\mathrm{SRW}}\left[T_{A}=\infty\right]
$$

observe that if $v_{j-1} \in A \backslash \partial A$ then $P_{v_{j-1}}^{\mathrm{SRW}}\left[T_{A}<\infty, S_{T_{A}}=v_{j}\right]$ simply equals $(2 d)^{-1}$, and $P_{v_{j-1}}^{\mathrm{SRW}}\left[T_{A}<\infty, S_{T_{A}}=v_{j}\right] \geq$ $(2 d)^{-1}$ in case $v_{i-1} \sim v_{i}$ (the inequality is strict if both $v_{i-1}$ and $v_{i}$ are on the boundary). For a finite sequence of traces $\mathbf{v}=\left(v^{(1)}, \ldots, v^{(|\mathbf{v}|)}\right)$ we define its total weight by

$$
\mathrm{P}[\mathbf{v}]=e^{-u \operatorname{cap}(A)} \frac{(u \operatorname{cap}(A))^{|\mathbf{v}|}}{|\mathbf{v}| !} \prod_{k=1}^{|\mathbf{v}|} \frac{e_{A}\left(v_{0}^{(k)}\right)}{\operatorname{cap}(A)} p_{v^{(k)}}
$$

(in fact, one may cancel $(\operatorname{cap}(A))^{|\mathbf{v}|}$ in the above formula, but we prefer to write it this way to make it clearer that the above equals the probability that $v^{(k)}$ 's are the traces left on $A$ by the trajectories of random interlacements ordered with respect to the $u$-coordinate). Write also

$$
L_{\mathbf{v}}(x)=\sum_{k, j} \mathbf{1}\left\{v_{j}^{(k)}=x\right\}
$$

so that $L_{\mathbf{v}}$ is the (total) local time of the traces of $\mathbf{v}$, and observe that

$$
\mathbf{P}\left[\mathcal{L}_{A}^{u}=\eta\right]=\sum_{\mathbf{v}: L_{\mathbf{v}}=\eta} \mathbf{P}[\mathbf{v}]
$$

Next, for $\mathbf{v}$ such that $L_{\mathbf{v}}\left(x_{0}\right) \geq 1$ let us define

$$
\mathbf{k}(\mathbf{v})=\min \left\{k: \text { there exists } j \text { such that } v_{j}^{k}=x_{0}\right\}
$$

and

$$
\mathbf{j}(\mathbf{v})=\min \left\{j: v_{j}^{(\mathbf{k}(\mathbf{v}))}=x_{0}\right\} .
$$

Also, for such $\mathbf{v}$ define a sequence of traces

$$
\hat{\mathbf{v}}=\left(v^{(1)}, \ldots, v^{(\mathbf{k}(\mathbf{v})-1)}, \hat{v}^{(\mathbf{k}(\mathbf{v}))}, v^{(\mathbf{k}(\mathbf{v})+1)}, \ldots, v^{(|\mathbf{v}|)}\right),
$$

where

$$
\hat{v}_{j}^{(\mathbf{k}(\mathbf{v}))}= \begin{cases}v_{j}^{(\mathbf{k}(\mathbf{v}))}, & \text { if } j \leq \mathbf{j}(\mathbf{v}) \\ y_{i}, & \text { if } j=\mathbf{j}(\mathbf{v})+i, 0<i \leq m, \\ v_{j-m}^{(\mathbf{k}(\mathbf{v}))}, & \text { if } j>\mathbf{j}(\mathbf{v})+m\end{cases}
$$

in words, $\hat{\mathbf{v}}$ is obtained from $\mathbf{v}$ by inserting the loop at the first possible location. Now, by construction, it holds that $p_{\hat{v}(\mathbf{k}(\mathbf{v}))}=(2 d)^{-m} p_{v^{(\mathbf{k}(\mathbf{v}))}}$ (since the whole path lies in $A \backslash \partial A$ ), and so $\mathrm{P}[\hat{\mathbf{v}}]=(2 d)^{-m} \mathrm{P}[\mathbf{v}]$. Now, $L_{\mathbf{v}}=\eta$ implies $L_{\hat{\mathbf{v}}}=\eta+\ell$ so, using (2.25) and the fact that $\mathbf{v} \mapsto \hat{\mathbf{v}}$ is an injection

$$
\mathbf{P}\left[\mathcal{L}_{A}^{u}=\eta+\ell\right] \geq \sum_{\mathbf{v}: L_{\mathbf{v}}=\eta} \mathbf{P}[\hat{\mathbf{v}}]=(2 d)^{-m} \mathbf{P}\left[\mathcal{L}_{A}^{u}=\eta\right]
$$

which concludes the proof of Lemma 2.8 . 
Let $A=\mathcal{C}_{M}(n+3 M \ln n)$ so that $Q\left(x^{\text {entry }}, M, n\right) \subset A$ for all $x \in \partial^{+} \mathcal{C}_{M}(n)$, and, for a fixed $\xi_{0} \in\{0,1\}^{\mathcal{C}_{M}(n)}$ define

$$
\begin{aligned}
\mathfrak{W}_{1}^{\xi_{0}}= & \left\{\eta \in\{0,1,2, \ldots\}^{A}: \mathcal{L}^{u}(x)=0 \text { for all } x \in Q\left(x^{\text {entry }}, M, n\right),\right. \\
& \left.\mathbf{1}\left\{\mathcal{L}^{u}(y) \geq 1\right\}=\mathbf{1}\left\{\xi_{0}(y)=1\right\} \text { for all } y \in \mathcal{I}_{\mathcal{C}_{M}(n)}^{u}\right\}
\end{aligned}
$$

and

$$
\begin{aligned}
\mathfrak{W}_{2}^{\xi_{0}}= & \left\{\eta \in\{0,1,2, \ldots\}^{A}: \mathcal{T}(x, M, n) \text { occurs on } \eta,\right. \\
& \left.\mathbf{1}\left\{\mathcal{L}^{u}(y) \geq 1\right\}=\mathbf{1}\left\{\xi_{0}(y)=1\right\} \text { for all } y \in \mathcal{I}_{\mathcal{C}_{M}(n)}^{u}\right\} .
\end{aligned}
$$

Next, let $\Phi$ be the set of local times $\ell$ of loops belonging to the event $E_{x, M, n}$, recall Lemma 2.4. Observe that, if $\eta \in \mathfrak{W}_{1}^{\xi_{0}}$ and $\ell \in \Phi$, then $\eta+\ell \in \mathfrak{W}_{2}^{\xi_{0}}$, and also if $\eta_{1}+\ell_{1}=\eta_{2}+\ell_{2}$ for $\eta_{1,2} \in \mathfrak{W}_{1}^{\xi_{0}}$ and $\ell_{1,2} \in \Phi$, then $\eta_{1}=\eta_{2}$ and $\ell_{1}=\ell_{2}$. With these observations, we write for any $\xi_{0}$ such that $\xi_{0}(0)>0$ and $\xi_{0}(x)>0$

$$
\begin{aligned}
\mathbf{P}\left[\mathcal{T}(x, M, n), \mathcal{I}_{\mathcal{C}_{M}(n)}^{u}=\xi_{0}\right] & =\sum_{\eta \in \mathfrak{W}_{2}^{\xi_{0}}} \mathbf{P}\left[\mathcal{L}_{A}^{u}=\eta\right] \\
& \geq \sum_{\eta^{\prime} \in \mathfrak{W}_{1}^{\xi_{0}}} \sum_{\ell \in \Phi} \mathbb{P}\left[\mathcal{L}_{A}^{u}=\eta^{\prime}+\ell\right] \\
& \geq \sum_{\eta^{\prime} \in \mathfrak{W}_{1}^{\xi_{0}}} \mathbb{P}\left[\mathcal{L}_{A}^{u}=\eta^{\prime}\right] \sum_{\ell \in \Phi}(2 d)^{-|\ell|} \\
& \geq n^{-\gamma / M} \mathbb{P}\left[Q\left(x^{\text {entry }}, M, n\right) \text { is vacant, } \mathcal{I}_{\mathcal{C}_{M}(n)}^{u}=\xi_{0}\right] \\
& \geq n^{-\gamma / M} \exp \left(-\frac{g_{M} \ln n}{\ln \ln n}\right) \mathbb{P}\left[\mathcal{I}_{\mathcal{C}_{M}(n)}^{u}=\xi_{0}\right],
\end{aligned}
$$

where the last inequality follows from Lemma 2.7 and the second-to-last one from Lemma 2.4. This implies that

$$
\mathbf{P}_{0}\left[\mathcal{T}(x, M, n) \mid \mathcal{I}_{\mathcal{C}_{M}(n)}^{u}, x \in \mathcal{I}_{\mathcal{C}_{M}(n)}^{u}\right] \geq n^{-\gamma / M},
$$

for any $x \in \partial^{+} \mathcal{C}_{M}(n)$

\section{Proofs of the main theorems}

\subsection{The biased random walk on the interlacement set in three dimensions has sub-polynomial speed}

In this section we prove Theorem 1.3.

Consider a sequence of cones $\mathcal{C}_{M}\left(j n^{1 / 3}\right), j=1, \ldots, n^{2 / 3}$, and let $\tau_{j}=T_{\partial \mathcal{C}_{M}\left(j n^{1 / 3}\right)}$, see Figure 3 . Recall the definition of the "good" environment from Lemma 2.7, and define a decreasing sequence of events

$$
\widehat{\mathcal{G}} k=\bigcap_{j=1}^{k} \bigcap_{x \in \partial^{+} \mathcal{C}_{M}\left(j n^{1 / 3}\right)} \mathcal{G}\left(x, M, j n^{1 / 3}\right)
$$

for $k \leq n^{2 / 3}$; let also $\widehat{\mathcal{G}}:=\widehat{\mathcal{G}}_{n^{2 / 3}}$. Observe that Lemma 2.7 implies that

$$
\mathbf{P}[\widehat{\mathcal{G}}] \geq 1-\sum_{k=1}^{n^{2 / 3}} M^{2}\left(j n^{1 / 3}\right)^{2} \times\left(j n^{1 / 3}\right)^{-12} \geq 1-C M^{2} n^{-3} .
$$




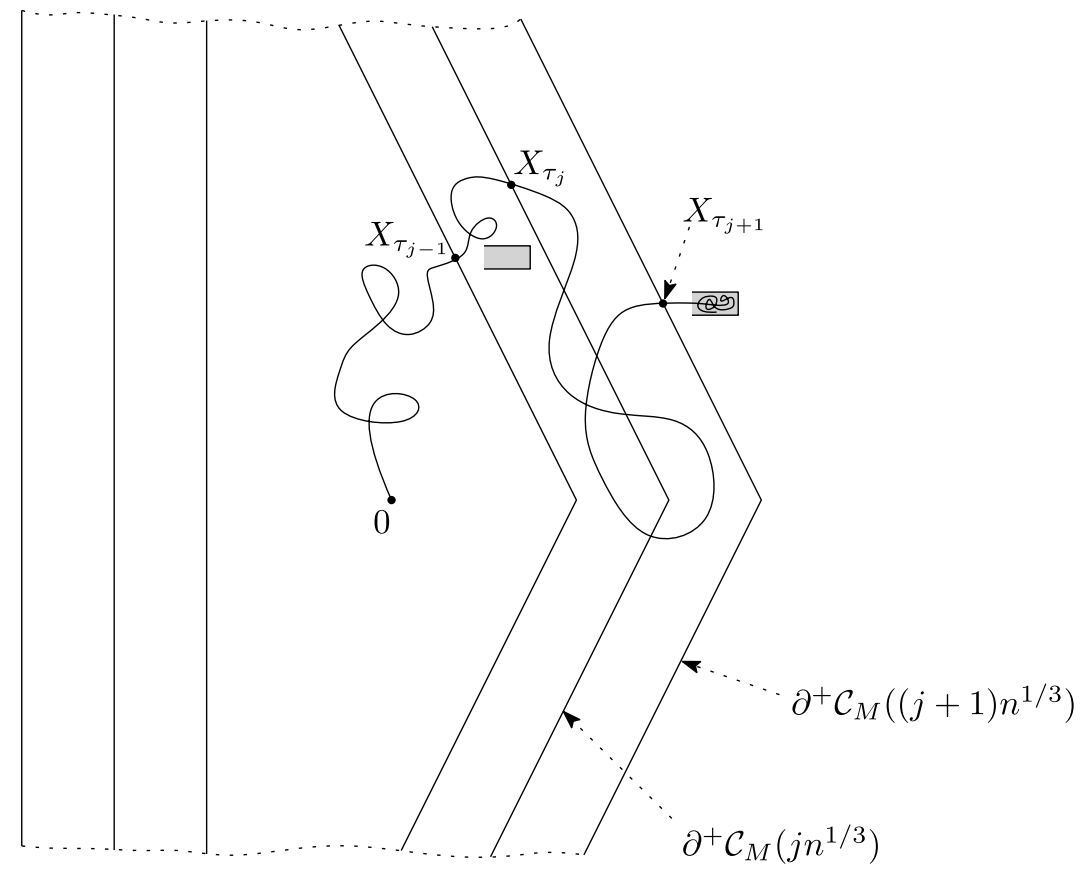

Fig. 3. On the proof of Theorem 1.3. There is a trap in front of $X_{\tau_{j-1}}$, but the walk manages to escape it; there is no trap in front of $X_{\tau_{j}}$; there is again a trap in front of $X_{\tau_{j+1}}$ and the walk finally gets caught there.

Next, let us define

$$
\zeta= \begin{cases}\infty, & \text { on } \widehat{\mathcal{G}} \\ j, & \text { on } \widehat{\mathcal{G}}_{j} \backslash \widehat{\mathcal{G}}_{j+1}, \text { if } j<n^{2 / 3}, \\ 0, & \text { on } \widehat{\mathcal{G}}_{1}^{\complement}\end{cases}
$$

and set formally $\tau_{0}=0, \tau_{\infty}=\infty$. We introduce another process $\widehat{X}$ in the following way: for $k \geq 0$

$$
\widehat{X}_{k}= \begin{cases}X_{k}, & \text { for } k \leq \tau_{\zeta} \\ X_{\tau_{\zeta}}, & \text { for } k>\tau_{\zeta}\end{cases}
$$

i.e., it is equal to the old process $X$ until the process stays in the "good" cone $\mathcal{C}_{M}\left(\zeta n^{1 / 3}\right)$, and then is stopped. We define also $\hat{\tau}_{j}=\widehat{T}_{\partial \mathcal{C}_{M}\left(j n^{1 / 3}\right)}$, where $\widehat{T}$ 's are the hitting times for $\widehat{X}$. Then, let us define a sequence of events

$$
\widehat{H}_{j}= \begin{cases}\Omega, & \text { on } \hat{\tau}_{j}=\infty \text { or when there exists } k \leq j \text { such that } \widehat{X}_{\hat{\tau}_{j}} \in \partial^{-} \mathcal{C}_{M}\left(k n^{1 / 3}\right), \\ \left\{\hat{\tau}_{j+1}-\hat{\tau}_{j}>n^{\frac{1}{3} M \ln \beta}\right\}, & \text { otherwise. }\end{cases}
$$

Let $\widehat{\mathcal{F}}_{\hat{\tau}_{j}}$ be the sigma-algebra generated by $\widehat{X}_{0}, \ldots, \widehat{X}_{\hat{\tau}_{j}}$.

We start by showing that when exiting a cone $\mathcal{C}_{M}\left(j n^{1 / 3}\right)$, conditionally on any type of past information which was likely to occur, we have a decent chance of spending a lot of time in $\mathcal{C}_{M}\left((j+1) n^{1 / 3}\right)$ :

Lemma 3.1. Fix any $M<\infty$ and $\beta>1$. We have for all $j=1, \ldots, n^{2 / 3}$

$$
\mathbb{P}\left[\widehat{H}_{j} \mid \widehat{\mathcal{F}}_{\hat{\tau}_{j}}\right] \geq n^{-2 \gamma / M},
$$

for all $n$ large enough. 
Proof. Assume that $x \in \partial^{+} \mathcal{C}_{M}\left(j n^{1 / 3}\right)$. In the following, we abbreviate $x^{\text {entry }}:=x^{\text {entry }}\left(x, M, j n^{1 / 3}\right)$ and $x^{\text {trap }}:=$ $x^{\text {trap }}\left(x, M, j n^{1 / 3}\right)$. On the event $\mathcal{T}\left(x, M, j n^{1 / 3}\right)$, we know that

(1) $x+k e_{1} \in \mathcal{I}_{u}$, for $0 \leq k \leq\left\lfloor\frac{3}{M} \ln n\right\rfloor$;

(2) $x^{\text {entry }}$ is $\mathcal{I}_{u}$-connected to $x^{\text {trap }}$ inside $Q\left(x^{\text {entry }}, M, j n^{1 / 3}\right)$;

(3) the connected component of $x^{\text {trap }}$ in $\mathcal{I}_{u} \backslash x^{\text {entry }}$ is finite.

In particular, the first property above implies

$$
\mathrm{P}_{x}^{\omega}\left[X_{1}=x+e_{1}, \ldots, X_{\left\lfloor\frac{3}{M} \ln n\right\rfloor}=x+\left\lfloor\frac{3}{M} \ln n\right\rfloor e_{1}\right] \geq\left(\frac{\beta}{\beta+2 d-1}\right)^{\left\lfloor\frac{3}{M} \ln n\right\rfloor} .
$$

Also on the event $\mathcal{T}\left(x, M, j n^{1 / 3}\right)$, by the second and third property above, we know that the connected component of $x^{\text {trap }}$ in $\mathcal{I}_{u} \backslash x^{\text {entry }}$ is finite and is adjacent to $x^{\text {entry }}$. We denote by $G$ the finite network formed by this connected component and $x^{\text {entry }}$. Let us also denote by $\mathrm{P}^{G}$ the probability for the walk restricted on $G$.

On $\mathcal{T}\left(x, M, j n^{1 / 3}\right)$ it is only possible to exit $Q\left(x^{\text {entry }}, M, j n^{1 / 3}\right)$ through $x^{\text {entry }}$; also, note that we defined traps in such a way that from $x$ entry the particle can jump only to the left or to the right, the jumps in the transversal directions cannot happen since the corresponding sites are not in the interlacement set. Hence, the jump from $x^{\text {entry }}$ to the right happens with probability $\frac{\beta}{\beta+1}$, and we can write

$$
\mathrm{P}_{x^{\text {entry }}}^{\omega}\left[T_{x^{\text {trap }}}<T_{Q\left(x^{\text {entry }}, M, j n^{1 / 3}\right)}\right]=\frac{\beta}{\beta+1} \mathrm{P}_{x^{\text {entry }}}^{G}\left[T_{x^{\text {trap }}}<T_{x^{\text {entry }}}\right] .
$$

Using the notation $\pi^{G}(x)=\sum_{y \in G} c(x, y)$ and $C^{G}$ for the effective conductance in $G$, we can use some standard facts about electrical network theory (see e.g. (2.4) in [11]) to obtain that

$$
\mathrm{P}_{x^{\text {entry }}}^{G}\left[T_{x^{\text {trap }}}<T_{x^{\text {entry }}}\right]=\frac{C^{G}\left(x^{\text {entry }} \leftrightarrow x^{\text {trap }}\right)}{\pi^{G}\left(x^{\text {entry }}\right)},
$$

and it is easy to see that $\pi^{G}\left(x^{\text {entry }}\right) \leq \gamma \beta^{x \cdot e_{1}+\left\lfloor\frac{3}{M} \ln n^{1 / 3}\right\rfloor}=\gamma \beta^{x \cdot e_{1}+\left\lfloor\frac{1}{M} \ln n\right\rfloor}\left(\right.$ recall that $x$ is a site on $\left.\partial^{+} \mathcal{C}_{M}\left(j n^{1 / 3}\right)\right)$. Furthermore, on $\mathcal{T}\left(x, M, j n^{1 / 3}\right)$, there is a simple path linking $x^{\text {entry }}$ to $x^{\text {trap }}$ of length at most $\gamma(M \ln n)^{3}$ edges all with conductances at least $\beta^{x \cdot e_{1}+\left\lfloor\frac{1}{M} \ln n\right\rfloor}$. Rayleigh's monotonicity principle then implies that

$$
C^{G}\left(x^{\text {entry }} \leftrightarrow x^{\text {trap }}\right) \geq \frac{\beta^{x \cdot e_{1}+\left\lfloor\frac{1}{M} \ln n\right\rfloor}}{\gamma(M \ln n)^{3}},
$$

so that

$$
\mathrm{P}_{x^{\text {entry }}}^{G}\left[T_{x^{\text {trap }}}<T_{x^{\text {entry }}}\right] \geq \gamma(M \ln n)^{-3} .
$$

The previous inequality along with (3.4) and (3.5) imply that on $\mathcal{T}\left(x, M, j n^{1 / 3}\right)$

$$
\mathrm{P}_{x}^{\omega}\left[T_{x^{\text {trap }}}<T_{\partial \mathcal{C}_{M}\left((j+1) n^{1 / 3}\right)}\right] \geq n^{-\gamma / M} .
$$

Moreover, on $\mathcal{T}\left(x, M, j n^{1 / 3}\right)$, we know that starting from $x^{\text {trap }}$ we need to reach $x^{\text {entry }}$ before we can exit $\mathcal{C}_{M}((j+$ 1) $\left.n^{1 / 3}\right)$ ). Furthermore, we see by reversibility that

$$
\mathrm{P}_{x^{\text {trap }}}^{\omega}\left[T_{x^{\text {entry }}}<T_{x^{\text {trap }}}\right]=\frac{\pi^{G}\left(x^{\text {entry }}\right)}{\pi^{G}\left(x^{\text {trap }}\right)} \mathrm{P}_{x^{\text {entry }}}^{\omega}\left[T_{x^{\text {trap }}}<T_{x^{\text {entry }}}\right] \leq \gamma \beta^{-\frac{M}{3} \ln n},
$$

so the number of returns to $x^{\text {trap }}$ before exiting $\left.\mathcal{C}_{M}\left((j+1) n^{1 / 3}\right)\right)$ is a geometric random variable of parameter at most $\gamma \beta^{-\frac{M}{3} \ln n}$. This means that the time to exit $\mathcal{C}_{M}\left((j+1) n^{1 / 3}\right)$ is larger than a geometric random variable of parameter at most $\gamma \beta^{-\frac{M}{3} \ln n}$, so for some uniformly positive $\varepsilon$ we have

$$
\mathrm{P}_{x^{\text {trap }}}^{\omega}\left[T_{\partial \mathcal{C}_{M}\left((j+1) n^{1 / 3}\right)} \geq \beta^{M \ln n}\right]>\varepsilon>0 .
$$


This result along with (3.6) and the Markov property implies that on any environment belonging to $\mathcal{T}\left(x, M, j n^{1 / 3}\right)$

$$
\mathrm{P}_{x}^{\omega}\left[T_{\partial \mathcal{C}_{M}\left((j+1) n^{1 / 3}\right)} \geq \beta^{M \ln n}\right] \geq n^{-\gamma / M} .
$$

Having dealt with the quenched probabilities, we move on. For any finite sequence $\tilde{x}=\left(x_{0}, x_{1}, \ldots, x_{m}\right)$ of sites in $\mathbb{Z}^{3}$ define

$$
\Gamma_{\omega}(\tilde{x})=\mathrm{P}_{x_{0}}^{\omega}\left[\widehat{X}_{1}=x_{1}, \ldots, \widehat{X}_{m}=x_{m}\right] .
$$

Let $x_{1}, \ldots, x$ be a sequence of sites in $\mathcal{C}_{M}\left(j n^{1 / 3}\right)$, and assume that $x \in \partial^{+} \mathcal{C}_{M}\left(j n^{1 / 3}\right)$. Write

$$
\begin{aligned}
\mathbb{P} & \left.\widehat{H}_{j} \mid \widehat{X}_{1}=x_{1}, \ldots, \widehat{X}_{\tau_{j}}=x\right] \\
& =\frac{\mathbf{E}_{0}\left(\mathrm{P}_{0}^{\omega}\left[\widehat{H}_{j} \mid \widehat{X}_{1}=x_{1}, \ldots, \widehat{X}_{\tau_{j}}=x\right] \Gamma_{\omega}\left(0, x_{1}, \ldots, x\right)\right)}{\mathbf{E}_{0} \Gamma_{\omega}\left(0, x_{1}, \ldots, x\right)} \\
& =\frac{\mathbf{E}_{0}\left(\mathrm{P}_{x}^{\omega}\left[\widehat{H}_{j}\right] \Gamma_{\omega}\left(0, x_{1}, \ldots, x\right)\right)}{\mathbf{E}_{0} \Gamma_{\omega}\left(0, x_{1}, \ldots, x\right)} \\
& \geq \frac{\mathbf{E}_{0}\left(\mathrm{P}_{x}^{\omega}\left[\widehat{H}_{j}\right] \mathbf{1}\left\{\omega \in \mathcal{T}\left(x, M, j n^{1 / 3}\right)\right\} \Gamma_{\omega}\left(0, x_{1}, \ldots, x\right)\right)}{\mathbf{E}_{0} \Gamma_{\omega}\left(0, x_{1}, \ldots, x\right)} .
\end{aligned}
$$

Note the following general fact: if $\xi, \eta \geq 0$ are random variables, $\eta$ is measurable with respect to a sigma-algebra $\mathcal{A}$, and $\mathbf{E}(\xi \mid \mathcal{A}) \geq \gamma_{1}$, then $\mathbf{E}(\xi \eta) \geq \gamma_{1} \mathbf{E} \eta$. Let $\mathcal{A}_{A}^{u}$ be the sigma-algebra generated by the random interlacements of level $u$ on the set $A \subset \mathbb{Z}^{3}$. Inequalities (2.26) and (3.7) imply that

$$
\mathbf{E}\left(\mathrm{P}_{x}^{\omega}\left[\widehat{H}_{j}\right] \mathbf{1}\left\{\omega \in \mathcal{T}\left(x, M, j n^{1 / 3}\right)\right\} \mid \mathcal{A}_{\mathcal{C}_{M}\left(j n^{1 / 3}\right)}^{u}\right) \geq n^{-2 \gamma / M},
$$

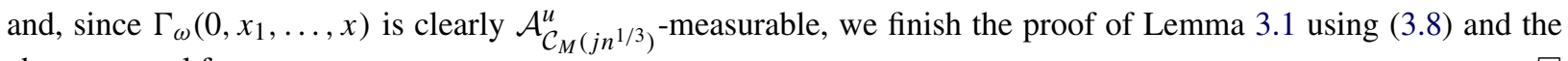
above general fact.

Lemma 3.2. There exists $\gamma_{1}>0$ such that

$$
\mathbb{P}\left[T_{\partial \mathcal{C}_{M}(n)} \geq n^{\frac{1}{3} M \ln \beta}\right] \geq 1-n^{-\gamma_{1}} .
$$

Proof. First, observe that Lemma 3.1 implies that

$$
\mathbb{P}\left[\bigcap_{j=1}^{n^{2} / 3} \widehat{H}_{j}^{\complement}\right] \leq \exp \left(-\gamma^{\prime} n^{\frac{2}{3}-\frac{\gamma}{M}}\right)
$$

(indeed, we have $n^{2 / 3}$ tries with success probability at least $n^{-\gamma / M}$, independently of the past).

Now, recalling the notation of $\Phi_{n}$ at 2.8 , write

$$
\begin{aligned}
& \mathbb{P}\left[T_{\partial \mathcal{C}_{M}(n)}<n^{\frac{1}{3} M \ln \beta}\right] \leq \mathbb{P}\left[T_{\partial \mathcal{C}_{M}(n)}<n^{\frac{1}{3} M \ln \beta}, \Phi_{n}\right]+\mathbb{P}\left[\Phi_{n}^{\complement}\right] \\
& \leq \mathbf{E}\left(\mathrm{P}^{\omega}\left[T_{\partial \mathcal{C}_{M}(n)}<n^{\frac{1}{3} M \ln \beta}, \Phi_{n}\right] \mathbf{1}\{\widehat{\mathcal{G}}\}\right)+\mathbf{P}\left[\widehat{\mathcal{G}}^{\complement}\right]+\mathbb{P}\left[\Phi_{n}^{\complement}\right] \\
& =\mathbf{E}\left(\mathrm{P}^{\omega}\left[\bigcap_{j=1}^{n^{2 / 3}} \widehat{H}_{j}^{\complement}\right] \mathbf{1}\{\widehat{\mathcal{G}}\}\right)+\mathbf{P}\left[\widehat{\mathcal{G}}^{\complement}\right]+\mathbb{P}\left[\Phi_{n}^{\complement}\right] \\
& \leq \mathbb{P}\left[\bigcap_{j=1}^{n^{2 / 3}} \widehat{H}_{j}^{\complement}\right]+\mathbf{P}\left[\widehat{\mathcal{G}}^{\complement}\right]+\mathbb{P}\left[\Phi_{n}^{\complement}\right],
\end{aligned}
$$




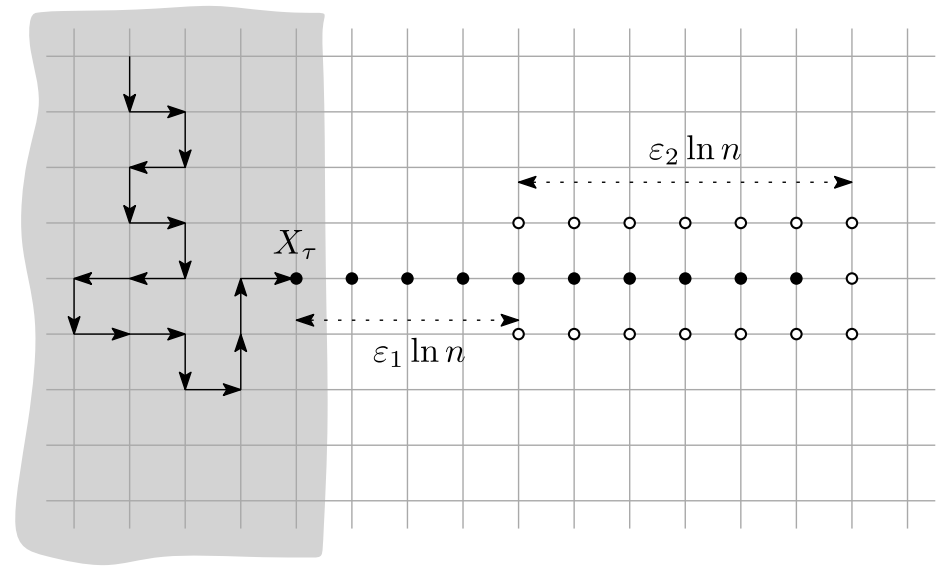

Fig. 4. Traps in higher dimensions ( $\varepsilon_{1,2}$ are supposed to be small enough). The shaded area corresponds to the already explored part of the environment; $\bullet$ 's belong to the interlacement set, and o's are vacant (the states of other sites can be arbitrary).

and we use (2.9), (3.2), and (3.9) to conclude the proof of Lemma 3.2.

We now finish the proof of Theorem 1.3. Indeed, Lemma 3.2 together with Borel-Cantelli's lemma imply that

$$
\mathbb{P}\left[T_{\partial \mathcal{C}_{M}\left(2^{k}\right)} \geq 2^{\frac{k}{3} M \ln \beta} \text { for almost all } k\right]=1 .
$$

So, for all large enough $t \in\left[2^{\frac{k}{3} M \ln \beta}, 2^{\frac{k+1}{3} M \ln \beta}\right.$ ) we have $X_{t} \in \mathcal{C}_{M}\left(2^{k+1}\right)$. Since $y \in \mathcal{C}_{M}(n)$ implies $\|y\| \leq M n$, we obtain

$$
\limsup _{t \rightarrow \infty} \frac{\ln \left\|X_{t}\right\|}{\ln t} \leq \limsup _{k \rightarrow \infty} \frac{(k+1) \ln 2+\ln M}{\frac{1}{3} k M \ln \beta \ln 2}=\frac{3}{M \ln \beta} \quad \mathbb{P} \text {-a.s., }
$$

which proves Theorem 1.3 since $M$ is arbitrary.

\subsection{Dimension $d \geq 4$}

In this section we prove Theorem 1.4.

The proof of this result is very similar to the proof of Theorem 1.3, so we only indicate where changes have to be made. The point is that, in dimensions $d \geq 4$, it costs too much to have a trap as on Figure 2. Instead, we use a simpler kind of traps, see Figure 4. When the particle faces a yet unexplored region, we just ask that there is a straight segment of sites belonging to the interlacement set of length $\ln n$ times a small constant, and the rightmost part of this segment is surrounded by vacant sites, as shown on the picture. It can be shown that the capacity of the "quiver" of the vacant sites is approximately $\gamma \varepsilon_{2} \ln n$, so the cost of having this quiver in the environment is roughly $n^{-\gamma^{\prime} \varepsilon_{2}}$, that is, power in $n$, but with a small power. Then, it can be easily seen that the cost of having the straight segment of occupied sites is similar, roughly $n^{-\gamma^{\prime \prime}\left(\varepsilon_{1}+\varepsilon_{2}\right)}$. Also, the decoupling argument works even better because of the $s^{d-2}$ in Proposition 2.6. So, it does not cost more than $n^{-\gamma^{\prime \prime}\left(\varepsilon_{1}+\varepsilon_{2}\right)}$ to have a trap like this each time when the particle faces the unexplored region. Now, regardless of our choice of $\varepsilon_{1,2}$, it is clear that if $\beta$ is very large, then the walk will spend a lot of time (say, at least $n^{2}$ ) in the trap with probability at least $n^{-\gamma_{1}}$, where $\gamma_{1}$ can be made arbitrarily small by decreasing $\varepsilon_{1,2}$. This shows the result.

\section{Acknowledgements}

Part of this work was done during the visit of the first author to IMECC-UNICAMP. Both authors thank the referee for careful reading of the manuscript and valuable comments and suggestions. 


\section{References}

[1] C. Alves and S. Popov. Conditional decoupling of random interlacements, 2015. Available at arXiv:1508.03405.

[2] G. Ben Arous and A. Fribergh. Biased random walks on random graphs, 2014. Available at arXiv:1406.5076.

[3] N. Berger and M. Biskup. Quenched invariance principle for simple random walks on percolation clusters. Probab. Theory Related Fields 130 (1-2) (2007) 83-120. MR2278453

[4] N. Berger, N. Gantert and Y. Peres. The speed of biased random walk on percolation clusters. Probab. Theory Related Fields 126 (2) (2003) 221-242. MR1990055

[5] J. Cerný and S. Popov. On the internal distance in the interlacement set. Electron. J. Probab. 17, paper No. 29 (2012) 1-25. MR2915665

[6] J. Cerný and A. Teixeira. From random walk trajectories to random interlacements. In Ensaios Matemáticos [Mathematical Surveys] 23. Sociedade Brasileira de Matemática, Rio de Janeiro, 2012. MR3014964

[7] A. Drewitz, B. Ráth and A. Sapozhnikov. An Introduction to Random Interlacements. Springer, Berlin, 2014. MR3308116

[8] A. Fribergh and A. Hammond. Phase transition for the speed of the biased random walk on a supercritical percolation cluster. Comm. Pure Appl. Math. 67 (2) (2014) 173-245. MR3149843

[9] G. Lawler. Intersections of Random Walks. Probability and Its Applications. Birkhäuser, Boston, 1991. MR1117680

[10] G. Lawler and V. Limic. Random Walk: A Modern Introduction. Cambridge Studies in Advanced Mathematics 123. Cambridge University Press, Cambridge, 2010. MR2677157

[11] R. Lyons and Y. Peres. Probability on Trees and Networks. Cambridge University Press, Cambridge, 2016. MR3616205

[12] P. Mathieu and A. Piatnitski. Quenched invariance principles for random walks on percolation clusters. Proc. Roy. Soc. Edinburgh Sect. A 463 (2007) 2287-2307. MR2345229

[13] S. Popov and A. Teixeira. Soft local times and decoupling of random interlacements. J. Eur. Math. Soc. (JEMS) 17 (10) (2015) $2545-2593$. MR3420516

[14] E. Procaccia, R. Rosenthal and A. Sapozhnikov. Quenched invariance principle for simple random walk on clusters in correlated percolation models. Probab. Theory Related Fields 166 (3) (2016) 619-657. MR3568036

[15] V. Sidoravicius and A.-S. Sznitman. Quenched invariance principles for walks on clusters of percolation or among random conductances. Probab. Theory Related Fields 129 (2) (2004) 219-244. MR2063376

[16] F. Spitzer. Principles of Random Walk. Springer, New York, 1976. MR0388547

[17] F. Stern. Conditional expectation of the duration in the classical ruin problem. Math. Mag. 48 (4) (1975) 200-203. MR0378106

[18] A.-S. Sznitman. On the anisotropic random walk on the percolation cluster. Comm. Math. Phys. 240 (1-2) (2003) 123-148. MR2004982

[19] A.-S. Sznitman. Topics in random walks in random environment. In School and Conference on Probability Theory 203-266. ICTP Lecture Notes Series, Trieste 17, 2004. MR2198849

[20] A.-S. Sznitman. Random motions in random media. In Mathematical Statistical Mechanics 219-242. Elsevier, Amsterdam, 2006. MR2581885

[21] A.-S. Sznitman. Vacant set of random interlacements and percolation. Ann. Math. (2) 171 (3) (2010) 2039-2087. MR2680403

[22] O. Zeitouni. Random walks in random environment. In XXXI summer school in probability, St Flour 193-312. Lecture Notes in Math. 1837, 2001. MR2071631 